\title{
Efficient Storage and Screening System for Onion BAC Clones
}

\author{
Go Suzuki*, Geum Sook Do and Yasuhiko Mukai \\ Division of Natural Science, Osaka Kyoiku University, 4-698-1 Asahigaoka, Kashiwara, Osaka 582-8582, Japan
}

Key Words: Allium cepa, bacterial artificial chromosome, PCR screening, pooled library.

Onion (Allium cepa L.) is an agriculturally important plant, and the genome of onion has been extensively studied at a conventional cytogenetical level. However, analysis of genomic organization in onion is very difficult because of the large genome size, ca. 15,000 Mb per haploid genome, a value about 100 times that of Arabidopsis thaliana (Arumuganathan and Earle 1991). The use of a bacterial artificial chromosome (BAC) vector, by which up to 300-kb fragment can be cloned, is a powerful tool for detailed analysis of complex genomes (Shizuya et al. 1992, Monaco and Larin 1994), especially for genomic analysis of plants with a large genome, such as onion and wheat. Up to now, the construction of a useful TAC (Transformation-competent Artificial Chromosome) library of common wheat, Triticum aestivum, had been reported (Liu et al. 2000), whereas a BAC library of onion has not yet been available. Construction and storage of a BAC library for such a large genome are laborious, and a large space in freezers is necessary for storage. In the case of an onion BAC library, 450,000 clones with $100-\mathrm{kb}$ inserts are necessary to cover $95 \%$ of the total genome, indicating that $>$ four-thousands 96 -well plates or $>$ one-thousand 384well plates are necessary to store them individually. In small laboratories, it is difficult to store more than several thousand plates, and such a system is not convenient for personal use. To overcome these shortcomings, we tried to pool BAC clones. We constructed a partial BAC library of onion by using a pBeloBAC11 vector and E. coli strain DH10B (Suzuki et al. 2001); a total of 48,000 clones with an average insert size of $100 \mathrm{~kb}$, that corresponds to 0.32 genome equivalent, were pooled in 480 glycerol stocks as follows.

\section{Storage and DNA isolation of pooled BAC clones}

In this study, the 48,000 onion BAC clones were pooled into 480 glycerol stocks (corresponding to five 96well plates), each of which contained 100 independent clones (Fig. 1). To minimize the bias of the frequency of pooled clones during culture, ten colonies, which were similar in size, were picked up and inoculated into $800 \mu \mathrm{LB}$

Communicated by T. Sasaki

Received September 17, 2001. Accepted November 3, 2001.

*Corresponding author (e-mail: gsuzuki@cc.osaka-kyoiku.ac.jp) with $12.5 \mathrm{mg} / \mathrm{l}$ chloramphenicol $(\mathrm{Cm})$ in a $1.5 \mathrm{ml}$ tube. After shaking at $37^{\circ} \mathrm{C}$ for $16 \mathrm{~h}$, ten $800 \mu \mathrm{l}$ cultures were pooled together in a $15 \mathrm{ml}$ tube, resulting in one pool containing 100 clones. From this pool (100 clones in $8 \mathrm{ml}), 300 \mu \mathrm{l}$ was used for a glycerol stock stored in one well of a 96-well plate at $-80^{\circ} \mathrm{C}$, and the rest of the pool $(7.7 \mathrm{ml})$ was used to isolate BAC DNA by the standard alkali method for polymerase chain reaction (PCR) screening. Thus, five 96-well plates were sufficient for stocking the 48,000 BAC clones, and they occupied only a small space in a deep freezer, suggesting that the pooled BAC library is suitable for small laboratories.

Problem associated with growth in pooled BAC clones

The most serious problem for the storage of the pooled BAC clones is associated with the growth of the clones during amplification. In our storage system, there might be a

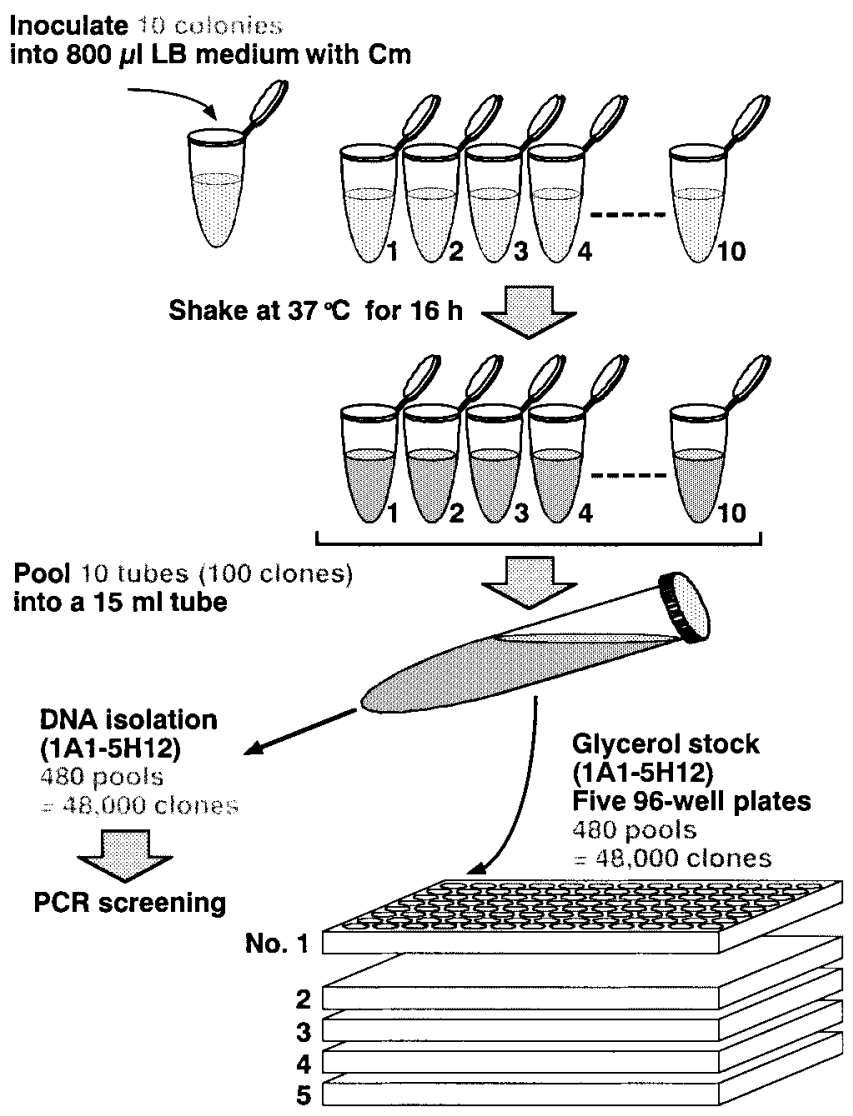

Fig. 1. Schematic representation of the stock and DNA isolation of pooled BAC clones. 
difference in the cell growth during overnight culture of each of the ten clones. In order to address this problem, we examined the frequency of the presence of the ten clones after bulked culture. Ten colonies similar in size were inoculated in $800 \mu \mathrm{l} \mathrm{LB}$ with $12.5 \mathrm{mg} / \mathrm{l} \mathrm{Cm}$ as in the case of the glycerol stock described above. The overnight culture, including 10 clones, was spread on an LB agar plate containing $12.5 \mathrm{mg} / \mathrm{l} \mathrm{Cm}$. BAC DNAs were isolated from $64 \mathrm{ran}-$ domly selected colonies, and characterized by HindIII digestion followed by $0.8 \%$ agarose gel electrophoresis. Two independent experiments were conducted, and the results are shown in Fig. 2. The first experiment was conducted for $1 \mathrm{a}$ to $1 \mathrm{j}$ clones, and the second experiment for $2 \mathrm{a}$ to $2 \mathrm{j}$ clones. Insert length of the clones was determined by pulsed field gel electrophoresis (PFGE) analysis with NotI-digested BAC DNA. None of the ten clones in each experiment were lost after the amplification, although the population of each clone in the culture was different among the clones (up to ca. 10-times difference), which was due to the competition among the clones during the amplification. Frequency of the existence of the clones was not related to their insert sizes. These results indicated that although the problem associated with the growth of these pooled BAC clones was detected in the overnight culture, no clones were lost, and a targeted clone could be screened from this stock.

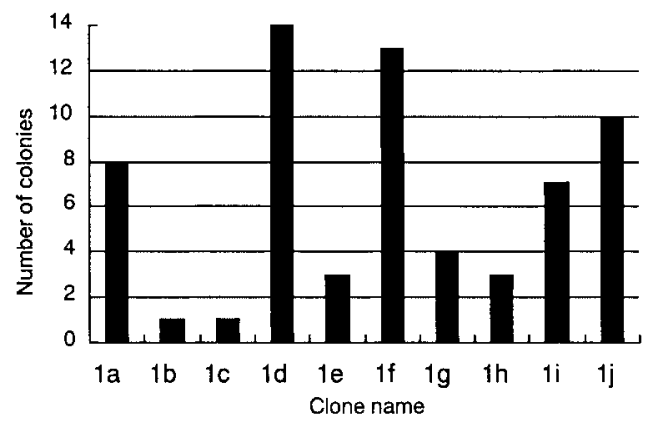

$1 \mathrm{a} \cdots 120 \mathrm{~kb}$

$1 \mathrm{~b}---114 \mathrm{~kb}$

$1 \mathrm{c} . \cdots 110 \mathrm{~kb}$

$1 d$--- 79 kb

$1 \mathrm{e}-\mathrm{-} 78 \mathrm{~kb}$

if $--63 \mathrm{~kb}$

$1 \mathrm{~g} \mathrm{---} 59 \mathrm{~kb}$

$1 \mathrm{~h}$--- $55 \mathrm{~kb}$

$1 i$... $47 \mathrm{~kb}$

1j -- $33 \mathrm{~kb}$

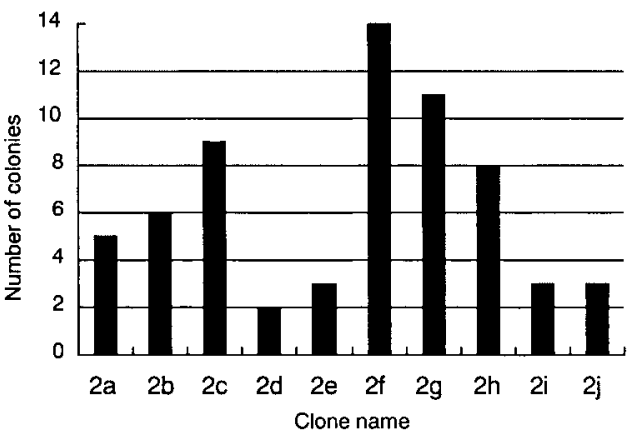

$2 \mathrm{a}---206 \mathrm{~kb}$

$2 \mathrm{~b}---115 \mathrm{~kb}$

2c --. $97 \mathrm{~kb}$

$2 \mathrm{~d} \ldots 79 \mathrm{~kb}$

$2 e$-.- $75 \mathrm{~kb}$

2f -- $68 \mathrm{~kb}$

$2 \mathrm{~g}--.66 \mathrm{~kb}$

$2 \mathrm{~h} \ldots 35 \mathrm{~kb}$

$2 i$... $32 \mathrm{~kb}$

2j -- $0 \mathrm{~kb}$

Fig. 2. Problem associated with growth in pooled BAC clones. Frequency of existence of the BAC clones after bulked culture of the ten clones was determined for two independent experiments (above, first experiment; below, second experiment). Insert length of each clone is shown on the right side of the graphs.

\section{PCR screening}

We tried to isolate $\mathrm{BAC}$ clones containing alliinase genes (VanDamme et al. 1992), to confirm the efficiency of the storage system. Each plate consisted of an array of 96 wells with 12 columns and 8 rows, and each well contained 100 clones, whose DNAs were isolated as BAC DNA pools, as described above. The BAC DNA pools were mixed using a three-dimensional (3D) scheme as superpools. For the first screening, 5 plate superpools (plates 1 to 5), 12 column superpools (wells 1 to 12 ), and 8 row superpools (wells A to $\mathrm{H}$ ) were used as templates of PCR amplification with alliinase-specific primers. PCR was performed with ExTaq DNA polymerase (TaKaRa), for 30 cycles of denaturation for $0.5 \mathrm{~min}$ at $94^{\circ} \mathrm{C}$, annealing for $0.5 \mathrm{~min}$ at $55^{\circ} \mathrm{C}$ and extension for $1 \mathrm{~min}$ at $72^{\circ} \mathrm{C}$, followed by a final extension for $5 \mathrm{~min}$, by using a DNA thermal cycler MP (TaKaRa). The first screening resulted in the identification of two positive bands (about $0.2 \mathrm{~kb}$ ) each in the plate superpools (plates 4 and 5), column superpools (wells 4 and 5), and row superpools (wells $\mathrm{F}$ and $\mathrm{G}$ ), indicating that there were more than two positive pools (Fig. 3a). For the second screening, PCR which was performed with the candidate pools (4F4, 4F5, $4 \mathrm{G} 4,4 \mathrm{G} 5,5 \mathrm{~F} 4,5 \mathrm{~F} 5,5 \mathrm{G} 4$, and 5G5) as templates, enabled to determine the positive pools, 4F4 and 5G5 (Fig. 3b). Subsequently, we screened the alliinase BAC clones by PCR from the two glycerol stocks corresponding to the 4F4 and 5G5

a

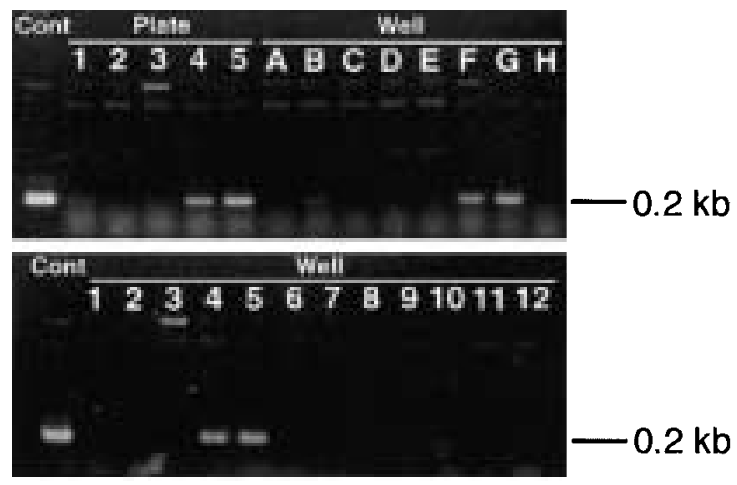

b

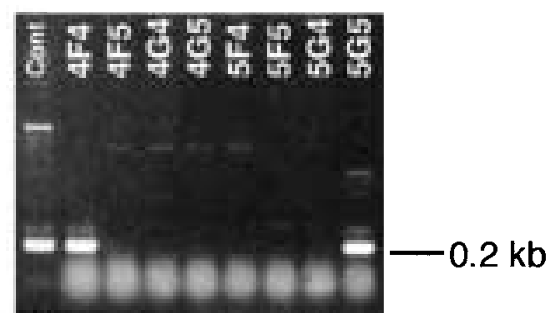

Fig. 3. Identification of the two pools containing alliinase genes by PCR screening from 3D superpools. (a) First screening for 3D superpools. (b) Second screening for candidate pools. The 0.2-kb PCR products of alliinase genes amplified from onion genomic DNA were loaded as positive controls (Cont). The primers used in this screening were designed from exon 3 of the onion alliinase gene (Accession No. L48614, Gilpin et al. 1995). 
pools, respectively, and two positive clones, 4F4-77 (96-kb insert) and 5G5-67 (105-kb insert), were successfully isolated (data not shown). Existence of the alliinase sequences in these BAC clones was confirmed by sequencing of the PCR fragments amplified from the clones. The alliinase BAC clones were identified from the partial library of 0.32 genome equivalent, because the alliinase genes form a multigene family in the Allium genome (VanDamme et al. 1992).

Recently, several reports have indicated the usefulness of the pooled BAC clones in plants; 300-600 colonies were pooled, and targeted sequences were successfully selected (Salimath and Bhattacharyya 1999, Liu et al. 2000). Ma et al. (2000) reported that up to 2000 BAC clones could be pooled and amplified without losing any target clones. In addition to these reports, the present study showed that the pooled BAC library of onion could be used for isolating multigene families and duplicated genomic regions. Molecular analysis of onion genome can be performed efficiently by using the BAC library, and complex gene arrangement embedded within repetitive sequences will be revealed in this plant with a large genome.

\section{Acknowledgments}

This work was supported in part by a grant from the Japan Society for the Promotion of Science (JSPS) under the Japan-Korea Joint Research Project, and a Grant-in-Aid for Scientific Research (B) (No. 09490024) from the Ministry of Education, Science, Sports and Culture, Japan.

\section{Literature Cited}

Arumuganathan,K. and E.D.Earle (1991) Nuclear DNA content of some important plant species. Plant Mol. Biol. Rep. 9: 208218.

Gilpin, B.J., D.W.Leung and J.E.Lancaster (1995) Nucleotide sequence of a nuclear clone of alliinase (Accession No. L48614) from onion (PGR95-125). Plant Physiol. 110: 336.

Liu, Y.-G., K.Nagaki, M.Fujita, K. Kawaura, M. Uozumi and Y. Ogihara (2000) Development of an efficient maintenance and screening system for large-insert genomic DNA libraries of hexaploid wheat in a transformation-competent artificial chromosome. Plant J. 23: 687-696.

Ma,Z., S. Weining, P.J.Sharp and C.Liu (2000) Non-gridded library: a new approach for BAC (bacterial artificial chromosome) exploitation in hexaploid wheat (Triticum aestivum). Nucl. Acids Res. 28: e106.

Monaco,A.P. and Z.Larin (1994) YACs, BACs, PACs and MACs: artificial chromosomes as research tools. Trend. Biotech. 12: 280-286.

Salimath,S.S. and M.K.Bhattacharyya (1999) Generation of a soybean BAC library, and identification of DNA sequences tightly linked to the Rpsl-k disease resistance gene. Theor. Appl. Genet. 98: 712-720.

Shizuya,H., B.Birren, U.-J.Kim, V.Mancino, T.Slepak, Y.Tachiiri and M.Simon (1992) Cloning and stable maintenance of 300kilobase-pair fragments of human DNA in Escherichia coli using an F-factor-based vector. Proc. Natl. Acad. Sci. USA 89: 8794-8797.

Suzuki, G., A.Ura, N.Saito, G.S.Do, B.B.Seo, M.Yamamoto and Y.Mukai (2001) BAC FISH analysis in Allium cepa. Genes Genet. Syst. 76: 251-255.

Van Damme,E.J.M., K.Smeets, S.Torrekens, F.Van Leuven and W.J. Peumans (1992) Isolation and characterization of alliinase cDNA clones from garlic (Allium sativum L.) and related species. Eur. J. Biochem. 209: 751-757. 